\title{
Quasi-Exactly Solvable Jacobi Elliptic Potential
}

\author{
Ancilla Nininahazwe \\ Institut de Pédagogie Appliquée, Université du Burundi, Bujumbura, Burundi \\ Email: nininaha@yahoo.fr
}

How to cite this paper: Nininahazwe, A. (2020) Quasi-Exactly Solvable Jacobi Elliptic Potential. Open Journal of Microphysics, 10, 21-33. https://doi.org/10.4236/ojm.2020.103003

Received: June 16, 2020

Accepted: July 17, 2020

Published: July 20, 2020

Copyright (c) 2020 by author(s) and Scientific Research Publishing Inc. This work is licensed under the Creative Commons Attribution International License (CC BY 4.0).

http://creativecommons.org/licenses/by/4.0/

\begin{abstract}
A new example of $2 \times 2$-matrix quasi-exactly solvable (QES) Hamiltonian which is associated to a Jacobi elliptic potential is constructed. We compute algebraically three necessary and sufficient conditions with the QES analytic method for the Jacobi Hamiltonian to have a finite dimensional invariant vector space. The matrix Jacobi Hamiltonian is called quasi-exactly solvable.
\end{abstract}

\section{Keywords}

Jacobi Elliptic Potential, QES Analytic Method, Three QES Conditions

\section{Introduction}

In quantum mechanics, the goal consists in computing the eigenvalues of linear Hamiltonian. In most cases, the spectrum of the Hamiltonian cannot be calculated algebraically. However, in few cases, some of which have the eigenvalues found explicitly. This type of Hamiltonian is called exactly solvable.

In the last few years, a new class of Hamiltonians which is intermediate to exactly solvable and non-solvable Hamiltonians has been discovered: the quasi-exactly solvable operators, for which a finite part of the eigenvalues can be computed algebraically. Many examples of QES Hamiltonians are studied in [1]-[13].

In the Refs. [10] [11] [12] [13], the QES analytic method is applied in order to establish a set of three necessary and sufficient conditions for Hamiltonians to have finite dimensional invariant vector spaces.

In this paper, we apply the same QES analytic method established in the Refs. [10] [11] [12] [13] in order to construct a $2 \times 2$-matrix QES Hamiltonian which is associated to a Jacobi elliptic potential.

This paper is organized as follows: in Section 2, based on [10] [11] [12] [13], we briefly recall the QES analytic method used to investigate the quasi-exact solvability of $2 \times 2$-matrix operators. In Section 3 , along the same lines as in the 
[10] [11] [12] [13], we apply the QES analytic method in order to construct a new $2 \times 2$-matrix QES Hamiltonian depending on Jacobi elliptic potential. We will consider two values of the constant $\delta$ : the case $\delta=1$ and the case $\delta=2$. The interest results will be computed.

\section{QES Analytic Method}

Taking account to the same lines as in [10] [11] [12] [13], we recall a general method to check whether a $2 \times 2$-matrix differential operator $H$ (in a variable $x$ ) preserves a vector space whose components are polynomials.

Consider the $2 \times 2$-matrix Hamiltonian of the following form [10] [11] [12] [13]:

$$
H=\left(\begin{array}{cc}
-\frac{\mathrm{d}^{2}}{\mathrm{~d} x^{2}}+V_{11}(x) & x^{\delta} \\
x^{\delta^{\prime}} & -\frac{\mathrm{d}^{2}}{\mathrm{~d} x^{2}}+V_{22}(x)
\end{array}\right)
$$

where

$$
V(x)=\left(\begin{array}{cc}
V_{11}(x) & x^{\delta} \\
x^{\delta^{\prime}} & V_{22}(x)
\end{array}\right), \delta=0,1,2, \quad \delta^{\prime}=1-\delta, \quad V_{12}(x)=x^{\delta}, \quad V_{21}(x)=x^{\delta^{\prime}}
$$

$V(x)$ is the potential associated to the Hamiltonian $H$ given by this above relation (1).

A gauge transformation and a change of variable on the Hamiltonian $H$ lead to the following Hamiltonian called the gauge one

$$
\tilde{H}=\phi^{-1} H \phi
$$

which can be written in his components as follows

$$
\tilde{H}=\tilde{H}_{1}+\tilde{H}_{0}+\tilde{H}_{-1} .
$$

More precisely, the diagonal components of $\tilde{H}_{1}$ are differential operators and the off-diagonal components $\left(\tilde{H}_{1}\right)_{12}$ and $\left(\tilde{H}_{1}\right)_{21}$ are respectively proportional to $x^{\delta}$ and $x^{\delta^{\prime}}$. The operators $\tilde{H}_{0}$ and $\tilde{H}_{-1}$ have lower degrees in all their components than the corresponding components in $\tilde{H}_{1}$.

Note that the invariant vector space of the Hamiltonian $\tilde{H}$ has the following form [10] [11] [12] [13]:

$$
W=\left\{\left(\begin{array}{c}
p_{n} \\
q_{n}
\end{array}\right)\right\}, m=n-\delta+1 \text { and } n, m \in \mathrm{N} .
$$

In order to obtain the QES conditions for $\tilde{H}$, the generic vector of the above vector space is of the form

$$
\varphi=\left(\begin{array}{c}
\alpha_{0} x^{n}+\alpha_{1} x^{n-1} \\
\beta_{0} x^{n-\delta+1}+\beta_{1} x^{n-\delta}
\end{array}\right)
$$

where $\alpha_{i}, \beta_{i}(i=0,1)$ are complex parameters. As a consequence the $2 \times 2$ -matrices $M_{1}, \tilde{M}_{1}, M_{0}$ are defined by 


$$
\begin{aligned}
\tilde{H}_{1}\left(\begin{array}{c}
\alpha_{0} x^{n} \\
\beta_{0} x^{n-\delta+1}
\end{array}\right) & =\operatorname{diag}\left(\begin{array}{ll}
x^{n+1} & x^{n-\delta+2}
\end{array}\right) M_{1}\left(\begin{array}{c}
\alpha_{0} \\
\beta_{0}
\end{array}\right), \\
\tilde{H}_{1}\left(\begin{array}{c}
\alpha_{1} x^{n-1} \\
\beta_{1} x^{n-\delta}
\end{array}\right) & =\operatorname{diag}\left(\begin{array}{ll}
x^{n} & x^{n-\delta+1}
\end{array}\right) \tilde{M}_{1}\left(\begin{array}{l}
\alpha_{1} \\
\beta_{1}
\end{array}\right), \\
\tilde{H}_{0}\left(\begin{array}{c}
\alpha_{0} x^{n} \\
\beta_{0} x^{n-\delta+1}
\end{array}\right) & =\operatorname{diag}\left(\begin{array}{ll}
x^{n} & x^{n-\delta+1}
\end{array}\right) M_{0}\left(\begin{array}{l}
\alpha_{0} \\
\beta_{0}
\end{array}\right) .
\end{aligned}
$$

The three necessary and sufficient QES conditions for $\tilde{H}$ to have an invariant vector space are

1) $M_{1}\left(\begin{array}{l}\alpha_{0} \\ \beta_{0}\end{array}\right)=\left(\begin{array}{l}0 \\ 0\end{array}\right)$,

2) $\tilde{M}_{1}^{t}\left(\begin{array}{c}-\beta_{0} \\ \alpha_{0}\end{array}\right)=\left(\begin{array}{l}0 \\ 0\end{array}\right)$,

3) $M_{0}\left(\begin{array}{c}1 \\ \frac{\beta_{0}}{\alpha_{0}}\end{array}\right)=\Lambda\left(\begin{array}{c}1 \\ \frac{\beta_{0}}{\alpha_{0}}\end{array}\right)$.

In the next step, we will apply in a same lines of this QES analytic method in order to prove the quasi-exact solvability of the $2 \times 2$-matrix QES Hamiltonian associated to Jacobi Elliptic Potential.

\section{QES Jacobi Hamiltonian}

\subsection{Case $\delta=1$}

In this section, we apply the QES analytic method established in previous section to check whether the $2 \times 2$-matrix operator is quasi-exactly solvable. We consider the $2 \times 2$-matrix Hamiltonian depending on the Jacobi elliptic potential of the form [10] [11] [12] [13]:

$$
H(z)=-\frac{\mathrm{d}^{2}}{\mathrm{~d} z^{2}} 1_{2}+V_{D}+V_{I}
$$

with

$$
\begin{gathered}
V_{D}=\operatorname{sn}^{2} \operatorname{diag}\left(a_{1}, a_{2}\right)+\operatorname{diag}\left(b_{1}, b_{2}\right), \\
V_{D}=\left(\begin{array}{cc}
s n^{2} a_{1}+b & 0 \\
0 & s n^{2} a_{2}-b
\end{array}\right)
\end{gathered}
$$

where $1_{2}$ is the matrix identity, $a_{1}, a_{2}, b_{1}, b_{2}, \theta$ denote real constants and $V_{I}$ is symmetric off-diagonal matrix of the form

$$
V_{I}(z)=\left(\begin{array}{cc}
0 & \theta c n d n \\
\theta c n d n & 0
\end{array}\right)
$$

Note that the sum $V_{D}+V_{I}$ is the Jacobi elliptic potential associated to the previous Hamiltonian $H(z)$.

Using the following the gauge transformation, the gauge Hamiltonian is written as follows 


$$
\begin{aligned}
& \tilde{H}(z)=g^{-1} H(z) g, \\
& \tilde{H}(z)=\left(\begin{array}{ll}
\tilde{H}_{11} & \tilde{H}_{12} \\
\tilde{H}_{21} & \tilde{H}_{22}
\end{array}\right)
\end{aligned}
$$

where

$$
\begin{gathered}
\tilde{H}_{11}=-\frac{\mathrm{d}^{2}}{\mathrm{~d} z^{2}}-2 \frac{g_{1}^{\prime}}{g_{1}} \frac{\mathrm{d}}{\mathrm{d} z}-\frac{g_{1}^{\prime \prime}}{g_{1}}+a_{1} s n^{2}+b, \\
\tilde{H}_{12}=\theta d n^{2}, \\
\tilde{H}_{21}=\theta c n^{2}, \\
\tilde{H}_{22}=-\frac{\mathrm{d}^{2}}{\mathrm{~d} z^{2}}-2 \frac{g_{2}^{\prime}}{g_{2}} \frac{\mathrm{d}}{\mathrm{d} z}-\frac{g_{2}^{\prime \prime}}{g_{2}}+a_{2} s n^{2}-b
\end{gathered}
$$

and

$$
\begin{aligned}
& g=\left(\begin{array}{cc}
g_{1} & 0 \\
0 & g_{2}
\end{array}\right), \\
& g=\left(\begin{array}{cc}
c n & 0 \\
0 & d n
\end{array}\right)
\end{aligned}
$$

The relevant change of variable consists in posing $t=s n^{2}(z, k)$. Taking account to the reference [9] [11], the differential symbol $\frac{\mathrm{d}^{2}}{\mathrm{~d} z^{2}}$ has the following form

$$
\frac{\mathrm{d}^{2}}{\mathrm{~d} z^{2}}=4 t(1-t)\left(1-k^{2} t\right) \frac{\mathrm{d}^{2}}{\mathrm{~d} t^{2}}+2\left(3 k^{2} t^{2}-2\left(1+k^{2}\right) t+1\right) \frac{\mathrm{d}}{\mathrm{d} t}
$$

We recall that for generic values of $k$, the Jacobi functions obey the following relations [9] [11]:

$$
\begin{array}{ll}
c n^{2}+s n^{2}=1, & d n^{2}+k^{2} s n^{2}=1 \\
\frac{\mathrm{d}}{\mathrm{d} z} s n=c n d n, & \frac{\mathrm{d}}{\mathrm{d} z} s n^{2}=2 s n c n d n \\
\frac{\mathrm{d}}{\mathrm{d} z} c n=-s n d n, & \frac{\mathrm{d}}{\mathrm{d} z} d n=-k^{2} \text { sncn }
\end{array}
$$

The following identities are used to establish the gauge Hamiltonian (11) in the variable $t=s n^{2}(z, k)$ [9] [11]:

$$
\begin{array}{ccc}
g & \frac{g^{\prime \prime}}{g} \quad \frac{g^{\prime}}{g}(\text { sncndn }) \\
100 & 0 \\
\text { sn } \quad 2 k^{2} t-\left(1+k^{2}\right) & k^{2} t^{2}-\left(1+k^{2}\right) t+1 \\
\text { cn } & 2 k^{2} t-1 \quad k^{2} t^{2}-t \\
\text { dn } & 2 k^{2} t-k^{2} & k^{2} t^{2}-k^{2} t \\
\text { cndn } & 6 k^{2} t-\left(1+k^{2}\right) & 2 k^{2} t^{2}-\left(1+k^{2}\right) t
\end{array}
$$




$$
\begin{array}{ccc}
\text { sndn } & 6 k^{2} t-\left(1+4 k^{2}\right) & 2 k^{2} t^{2}-\left(1+2 k^{2}\right) t+1 \\
\text { sncn } & 6 k^{2} t-\left(4+k^{2}\right) & 2 k^{2} t^{2}-\left(2+k^{2}\right) t+1 \\
\text { sncndn } & 12 k^{2} t-4\left(1+k^{2}\right) & 3 k^{2} t^{2}-2\left(1+k^{2}\right) t+1
\end{array}
$$

Referring to the above relations (16), for $g_{1}=c n$, the second term and the third term of the operator $\tilde{H}_{11}$ of the Equation (12) are written as follows:

$$
\begin{gathered}
-2 \frac{g_{1}^{\prime}}{g_{1}} \frac{\mathrm{d}}{\mathrm{d} z}=-2 \frac{g_{1}^{\prime}}{g_{1}}(\text { sncndn }) \frac{d}{d t}, \\
-2 \frac{g_{1}^{\prime}}{g_{1}} \frac{\mathrm{d}}{\mathrm{d} z}=-4\left[k^{2} t^{2}-t\right] \frac{\mathrm{d}}{\mathrm{d} t}, \\
-\frac{g_{1}^{\prime \prime}}{g_{1}}=-2 k^{2} t+1 .
\end{gathered}
$$

Referring to the same identities given by the Equation (16), for $g_{2}=d n$, the second term and the third term of the operator $\tilde{H}_{22}$ of the Equation (12) are of the following form:

$$
\begin{gathered}
-2 \frac{g_{2}^{\prime}}{g_{2}} \frac{\mathrm{d}}{\mathrm{d} z}=-2 \frac{g_{2}^{\prime}}{g_{2}}(2 \text { sncndn }) \frac{\mathrm{d}}{\mathrm{d} t}, \\
-2 \frac{g_{2}^{\prime}}{g_{2}} \frac{\mathrm{d}}{\mathrm{d} z}=4\left(k^{2} t^{2}-k^{2} t\right) \frac{\mathrm{d}}{\mathrm{d} t}, \\
-\frac{g_{2}^{\prime \prime}}{g_{2}}=-2 k^{2} t+k^{2} .
\end{gathered}
$$

Considering the change of variable $t=s n^{2}(z, k)$, the fourth and fifth terms of the components $\tilde{H}_{11}$ and $\tilde{H}_{22}$ of the gauge Hamiltonian $\tilde{H}$ are respectively rewritten as follows:

$$
\left(\begin{array}{cc}
a_{1} s n^{2}+b & 0 \\
0 & a_{2} s n^{2}-b
\end{array}\right)=\left(\begin{array}{cc}
a_{1} t+b & 0 \\
0 & a_{2} t-b
\end{array}\right)
$$

Taking account of the change of variable $t=s n^{2}(z, k)$ the identities $c n^{2}+s n^{2}=1$ and $d n^{2}+k^{2} s n^{2}=1$ lead respectively to new form of the two off-diagonal components of the gauge Hamiltonian given by the Equation (12):

$$
\begin{gathered}
\tilde{H}_{12}=\theta d n^{2}, \\
\tilde{H}_{12}=\theta-\theta k^{2} t \\
\tilde{H}_{21}=\theta c n^{2}, \\
\tilde{H}_{21}=\theta-\theta t
\end{gathered}
$$

Replacing the terms of the components of the Hamiltonian $\tilde{H}$ given by the Equation (12) by the expressions (14) and (17)-(23), one can easily write (in variable $t$ ) the gauge Hamiltonian as follows

$$
\begin{aligned}
\tilde{H}_{11}= & -4 t(1-t)\left(1-k^{2} t\right) \frac{\mathrm{d}^{2}}{\mathrm{~d} t^{2}}-2\left[5 k^{2} t^{2}-2\left(2+k^{2}\right) t+1\right] \frac{\mathrm{d}}{\mathrm{d} t}, \\
& +a_{1} t-2 k^{2} t+b+1
\end{aligned}
$$




$$
\begin{gathered}
\tilde{H}_{12}=\theta\left(1-k^{2} t\right), \\
\tilde{H}_{21}=\theta(1-t), \\
\tilde{H}_{22}=-4 t(1-t)\left(1-k^{2} t\right) \frac{\mathrm{d}^{2}}{\mathrm{~d} z^{2}}-2\left[5 k^{2} t^{2}-2\left(1+2 k^{2}\right) t+1\right] \frac{\mathrm{d}}{\mathrm{d} t} . \\
+a_{2} t-2 k^{2} t+k^{2}-b
\end{gathered}
$$

The next step is to establish the QES conditions of the gauge Hamiltonian. In other words, we put out the expressions of the real parameters $a_{1}, b$ and $\theta$.

Let us express the gauge Hamiltonian $\tilde{H}$ given by the above relations (24) in its components according to

$$
\tilde{H}=\tilde{H}_{1}+\tilde{H}_{0}+\tilde{H}_{-1}
$$

where

$$
\begin{gathered}
\tilde{H}_{1}=\left(\begin{array}{cc}
-4 k^{2} t^{3} \frac{\mathrm{d}^{2}}{\mathrm{~d} t^{2}}-10 k^{2} t^{2} \frac{\mathrm{d}}{\mathrm{d} t}+\left(a_{1}-2 k^{2}\right) t & -\theta k^{2} t \\
-\theta t & -4 k^{2} t^{3} \frac{\mathrm{d}^{2}}{\mathrm{~d} t^{2}}-10 k^{2} t^{2} \frac{\mathrm{d}}{\mathrm{d} t}+\left(a_{2}-2 k^{2}\right) t
\end{array}\right), \\
\tilde{H}_{0}=\left(\begin{array}{cc}
\left(4 k^{2}+4\right) t^{2} \frac{\mathrm{d}^{2}}{\mathrm{~d} t^{2}}+4\left(2+k^{2}\right) t \frac{\mathrm{d}}{\mathrm{d} t}+1+b & \theta \\
\theta & \left(4 k^{2}+4\right) t^{2} \frac{\mathrm{d}^{2}}{\mathrm{~d} t^{2}}+4\left(1+2 k^{2}\right) t \frac{\mathrm{d}}{\mathrm{d} t}+k^{2}-b
\end{array}\right), \\
\tilde{H}_{-1}=\left(\begin{array}{cc}
-4 t \frac{\mathrm{d}^{2}}{\mathrm{~d} t^{2}}-2 \frac{\mathrm{d}}{\mathrm{d} t} & 0 \\
0 & -4 t \frac{\mathrm{d}^{2}}{\mathrm{~d} t^{2}}-2 \frac{\mathrm{d}}{\mathrm{d} t}
\end{array}\right)
\end{gathered}
$$

As $\delta=1$, the generic wave function $\psi$ of the gauge Hamiltonian given by the Equation (11) is written as follows

$$
\psi=\left(\begin{array}{c}
\alpha_{0} t^{n}+\alpha_{1} t^{n-1}+\cdots \\
\beta_{0} t^{n}+\beta_{1} t^{n-1}+\cdots
\end{array}\right)
$$

Note that the action of these above three gauge components of $\tilde{H}$ given by the relations (26) on the wave function $\psi$ given by the relation (27) leads to the following expressions:

$$
\begin{gathered}
\tilde{H}_{1}\left(\begin{array}{l}
t^{n} \\
t^{n}
\end{array}\right) \cong\left(\begin{array}{l}
t^{n+1} \\
t^{n+1}
\end{array}\right), \\
\tilde{H}_{0}\left(\begin{array}{l}
t^{n} \\
t^{n}
\end{array}\right) \cong\left(\begin{array}{c}
t^{n} \\
t^{n}
\end{array}\right), \\
\tilde{H}_{-1}\left(\begin{array}{c}
t^{n} \\
t^{n}
\end{array}\right) \cong\left(\begin{array}{l}
t^{n-1} \\
t^{n-1}
\end{array}\right) .
\end{gathered}
$$

After some algebraic manipulations, one can easily obtain the $2 \times 2$-matrices $M_{1}, \tilde{M}_{1}, M_{0}$ respectively as follows [9] [10] [11] [12]: 


$$
\begin{gathered}
\tilde{H}_{1}\left(\begin{array}{l}
\alpha_{0} t^{n} \\
\beta_{0} t^{n}
\end{array}\right)=\operatorname{diag}\left(t^{n+1}, t^{n+1}\right) M_{1}\left(\begin{array}{l}
\alpha_{0} \\
\beta_{0}
\end{array}\right), \\
\tilde{H}_{1}\left(\begin{array}{c}
\alpha_{1} t^{n-1} \\
\beta_{1} t^{n-1}
\end{array}\right)=\operatorname{diag}\left(t^{n}, t^{n}\right) \tilde{M}_{1}\left(\begin{array}{l}
\alpha_{1} \\
\beta_{1}
\end{array}\right), \\
\tilde{H}_{0}\left(\begin{array}{c}
\alpha_{0} t^{n} \\
\beta_{0} t^{n}
\end{array}\right)=\operatorname{diag}\left(t^{n}, t^{n}\right) M_{0}\left(\begin{array}{l}
\alpha_{0} \\
\beta_{0}
\end{array}\right) .
\end{gathered}
$$

Taking account to these above expressions given by the Equation (29), one can easily find the following matrices:

$$
\begin{gathered}
M_{1}=\left(\begin{array}{cc}
-4 k^{2} n(n-1)-10 n k^{2}+a_{1}-2 k^{2} & -\theta k^{2} \\
-\theta & -4 k^{2} n(n-1)-10 n k^{2}+a_{2}-2 k^{2}
\end{array}\right), \\
\tilde{M}_{1}=\left(\begin{array}{cc}
-4 k^{2} n(n-1)(n-2)-10 k^{2}(n-1)+a_{1}-2 k^{2} & -\theta k^{2} \\
-\theta & -4 k^{2} n(n-1)(n-2)-10 k^{2}(n-1)+a_{2}-2 k^{2}
\end{array}\right) \\
M_{0}=\left(\begin{array}{cc}
n(n-1)\left(4 k^{2}+4\right)+4\left(2+k^{2}\right) n+1+b & \theta \\
\theta & n(n-1)\left(4 k^{2}+4\right)+4\left(1+2 k^{2}\right) n+k^{2}-b
\end{array}\right) \cdot(30)
\end{gathered}
$$

The three necessary QES conditions for the operator $\tilde{H}$ to have a finite dimensional invariant vector space are successively obtained [10] [11] [12] [13]:

1) The first QES condition is

$$
\begin{gathered}
M_{1}\left(\begin{array}{l}
\alpha_{0} \\
\beta_{0}
\end{array}\right)=\left(\begin{array}{l}
0 \\
0
\end{array}\right), \\
\operatorname{det} M_{1}=0 \\
\theta^{2}=16 k^{2} n^{4}+48 k^{2} n^{3}+52 k^{2} n^{2}+24 k^{2} n+4 k^{4}+\left(-4 n^{2}-6 n-2\right)\left(a_{1}+a_{2}\right)+\frac{a_{1} a_{2}}{k^{2}}
\end{gathered}
$$

2) The second QES condition is as follows

$$
\begin{gathered}
\tilde{M}_{1}^{t}\left(\begin{array}{c}
-\beta_{0} \\
\alpha_{0}
\end{array}\right)=\left(\begin{array}{l}
0 \\
0
\end{array}\right), \\
\operatorname{det} \tilde{M}_{1}=0, \\
\operatorname{det} \tilde{M}_{1}=\left[-4 k^{2} n(n-1)(n-2)-10 k^{2}(n-1)+a_{1}-2 k^{2}\right] \\
\times\left[-4 k^{2} n(n-1)(n-2)-10 k^{2}(n-1)+a_{2}-2 k^{2}\right]-\theta^{2} k^{2}
\end{gathered}
$$

In this above equation replacing $\theta^{2}$ by its value (31) and after some algebraic manipulations, the second QES condition is obtained

$$
a_{1}=\frac{-64 k^{4} n^{3}+48 k^{4} n^{2}+24 k^{4} n+4 k^{4}-8 k^{2} n a_{2}-2 k^{2} a_{2}}{8 k^{2} n+2 k^{2}}
$$

3) The final and the third QES condition is computed as follows

$$
M_{0}\left(\begin{array}{c}
1 \\
\frac{\beta_{0}}{\alpha_{0}}
\end{array}\right)=\Lambda\left(\begin{array}{c}
1 \\
\frac{\beta_{0}}{\alpha_{0}}
\end{array}\right),
$$


where $\Lambda$ is a constant and

$$
\frac{\beta_{0}}{\alpha_{0}}=\frac{-4 k^{2} n^{2}-6 k^{2} n+a_{1}-2 k^{2}}{\theta k^{2}}
$$

Referring to the expression of the matrix $M_{0}$ given by the Equation (30) and the relation (34), the relation (33) leads to the following third QES condition:

$$
b=\frac{N}{D}
$$

where

$$
\begin{gathered}
N=-16 k^{2} n^{4}-32 k^{2} n^{3}-24 k^{2} n^{2}-16 k^{4} n^{3}-28 k^{4} n^{2}-10 k^{2} n-14 k^{4} n \\
+8 a_{1} n+8 a_{1} n^{2}+4 a_{1} k^{2} n+3 a_{1}+a_{1} k^{2}-\frac{a_{1}^{2}}{k^{2}}-2 k^{2}-2 k^{4}+\theta^{2} k^{2} \\
D=8 k^{2} n^{2}-12 k^{2} n+2 a_{1}-4 k^{2}
\end{gathered}
$$

Taking account to the QES conditions given by the Equations (31), (32) and (35), we can conclude that the operator $\tilde{H}$ (therefore $H$ ) is quasi-exactly solvable [10] [11] [12] [13]. In other words, a finite part of the spectrum of the operator $\tilde{H}$ can be computed algebraically.

\subsection{Case $\delta=2$}

Along the same lines applied for the previous case (i.e. for the case $\delta=1$ ), we perform a gauge transformation according to

$$
\begin{gathered}
\tilde{H}(z)=f^{-1} H(z) f, \\
\tilde{H}(z)=\left(\begin{array}{ll}
\tilde{H}_{11} & \tilde{H}_{12} \\
\tilde{H}_{21} & \tilde{H}_{22}
\end{array}\right)
\end{gathered}
$$

with

$$
\begin{gathered}
f=\left(\begin{array}{cc}
f_{1} & 0 \\
0 & f_{2}
\end{array}\right), f_{1}=s n, \quad f_{2}=\text { sncndn, } \\
f=\left(\begin{array}{cc}
s n & 0 \\
0 & \text { sncndn }
\end{array}\right), f^{-1}=\left(\begin{array}{cc}
\frac{1}{s n} & 0 \\
0 & \frac{1}{\text { sncndn }}
\end{array}\right),
\end{gathered}
$$

After some algebraic manipulations, the components of the above Hamiltonian $\tilde{H}$ are written as follows

$$
\begin{gathered}
\tilde{H}_{11}=-\frac{\mathrm{d}^{2}}{\mathrm{~d} z^{2}}-2 \frac{f_{1}^{\prime}}{f_{1}} \frac{\mathrm{d}}{\mathrm{d} z}-\frac{f_{1}^{\prime \prime}}{f_{1}}+a_{1} s n^{2}+b, \\
\tilde{H}_{12}=\theta c n^{2} d n^{2}, \\
\tilde{H}_{21}=\theta, \\
\tilde{H}_{22}=-\frac{\mathrm{d}^{2}}{\mathrm{~d} z^{2}}-2 \frac{f_{2}^{\prime}}{f_{2}} \frac{\mathrm{d}}{\mathrm{d} z}-\frac{f_{2}^{\prime \prime}}{f_{2}}+a_{2} s n^{2}-b
\end{gathered}
$$

and the operator $H(z)$ is given by the Equation (8). 
Referring to the table of identities given by the Equation (16), the second term and the third term of the component operator $\tilde{H}_{11}$ (38) are of the following form

$$
\begin{gathered}
-2 \frac{f_{1}^{\prime}}{f_{1}} \frac{\mathrm{d}}{\mathrm{d} z}=-4 \frac{f_{1}^{\prime}}{f_{1}}(\text { sncndn }) \frac{\mathrm{d}}{\mathrm{d} t}, \\
-2 \frac{f_{1}^{\prime}}{f_{1}} \frac{\mathrm{d}}{\mathrm{d} z}=-4\left[k^{2} t^{2}-\left(1+k^{2}\right) t+1\right] \frac{\mathrm{d}}{\mathrm{d} t}, \\
-\frac{f_{1}^{\prime \prime}}{f_{1}}=-2 k^{2} t+k^{2}+1
\end{gathered}
$$

with

$$
f_{1}=s n \text {. }
$$

For $f_{2}=s n c n d n$, taking account to the same identities (16), the following second and the third terms of the component operator $\tilde{H}_{22}$ (38):

$$
\begin{gathered}
-2 \frac{f_{2}^{\prime}}{f_{2}} \frac{\mathrm{d}}{\mathrm{d} z}=-4 \frac{f_{2}^{\prime}}{f_{2}}(\text { sncndn }) \frac{\mathrm{d}}{\mathrm{d} t}, \\
-2 \frac{f_{1}^{\prime}}{f_{1}} \frac{\mathrm{d}}{\mathrm{d} z}=-\left[12 k^{2} t^{2}-8\left(1+k^{2}\right) t+4\right] \frac{\mathrm{d}}{\mathrm{d} t}, \\
-\frac{f_{2}^{\prime \prime}}{f_{2}}=-12 k^{2} t+4\left(1+k^{2}\right) .
\end{gathered}
$$

Referring to the change of variable $t=s n^{2}(z, k)$, the fourth and fifth terms of the components $\tilde{H}_{11}$ and $\tilde{H}_{22}$ of the gauge Hamiltonian $\tilde{H}$ given by the relation (38) have the following form:

$$
\left(\begin{array}{cc}
a_{1} s n^{2}+b & 0 \\
0 & a_{2} s n^{2}-b
\end{array}\right)=\left(\begin{array}{cc}
a_{1} t+b & 0 \\
0 & a_{2} t-b
\end{array}\right)
$$

Taking account to the change of the variable $t=s n^{2}(z, k)$ and the relations $c n^{2}+s n^{2}=1, d n^{2}+k^{2} s n^{2}=1$, the off-diagonal component $\tilde{H}_{12}$ of the gauge Hamiltonian $\tilde{H}$ given by the relation (38) is written as the follows

$$
\begin{gathered}
\tilde{H}_{12}=\theta c n^{2} d n^{2}, \\
\tilde{H}_{12}=\theta(1-t)\left(1-k^{2} t\right)
\end{gathered}
$$

Note that the off-diagonal component $\tilde{H}_{21}$ of the gauge Hamiltonian $\tilde{H}$ given by the relation (38) keeps the same expression

$$
\tilde{H}_{21}=\theta
$$

Replacing the four components of the gauge Hamiltonian $\tilde{H}(t)$ given by the Equation (38) by their expressions (14) and (39)-(45), one can easily found their final form in variable $t$.

$$
\begin{aligned}
\tilde{H}_{11}= & -4 t \frac{\mathrm{d}^{2}}{\mathrm{~d} t^{2}}+4\left(k^{2}+1\right) t^{2} \frac{\mathrm{d}^{2}}{\mathrm{~d} t^{2}}-4 k^{2} t^{3} \frac{\mathrm{d}^{2}}{\mathrm{~d} t^{2}}-6 k^{2} t^{2} \frac{\mathrm{d}}{\mathrm{d} t}+\left(4-4 k^{2}\right) t \frac{\mathrm{d}}{\mathrm{d} t} \\
& +\left(2+4 k^{2}\right) \frac{\mathrm{d}}{\mathrm{d} t}+\left(a_{1}-2 k^{2}\right) t+1+k^{2}+b
\end{aligned}
$$




$$
\begin{gathered}
\tilde{H}_{12}=\theta k^{2} t^{2}-\theta\left(k^{2}+1\right) t+\theta, \\
\tilde{H}_{21}=\theta, \\
\tilde{H}_{22}=-4 t \frac{\mathrm{d}^{2}}{\mathrm{~d} t^{2}}+4\left(k^{2}+1\right) t^{2} \frac{\mathrm{d}^{2}}{\mathrm{~d} t^{2}}-4 k^{2} t^{3} \frac{\mathrm{d}^{2}}{\mathrm{~d} t^{2}}-18 k^{2} t^{2} \frac{\mathrm{d}}{\mathrm{d} t} \\
+\left(12+12 k^{2}\right) t \frac{\mathrm{d}}{\mathrm{d} t}-6 \frac{\mathrm{d}}{\mathrm{d} t}+\left(a_{2}-12 k^{2}\right) t+4+4 k^{2}-b
\end{gathered}
$$

Note that the generic element of the invariant vector space $V$ under the action of the gauge operator $\tilde{H}(t)$ is given by the Equation (5) as in the QES analytic method:

$$
\begin{gathered}
\psi=\left(\begin{array}{c}
\alpha_{0} t^{n}+\alpha_{1} t^{n-1} \\
\beta_{0} t^{n-\delta+1}+\beta_{1} t^{n-\delta}
\end{array}\right), \\
\psi=\left(\begin{array}{c}
\alpha_{0} t^{n}+\alpha_{1} t^{n-1} \\
\beta_{0} t^{n-1}+\beta_{1} t^{n-2}
\end{array}\right) .
\end{gathered}
$$

with $\delta=2$.

Note that the action of the gauge components of $\tilde{H}$ given by the relations (46) on the generic function $\psi$ given by the relation (47) leads to the following expressions:

$$
\begin{aligned}
& \tilde{H}_{1}\left(\begin{array}{c}
t^{n} \\
t^{n-1}
\end{array}\right) \cong\left(\begin{array}{c}
t^{n+1} \\
t^{n}
\end{array}\right), \\
& \tilde{H}_{0}\left(\begin{array}{c}
t^{n} \\
t^{n-1}
\end{array}\right) \cong\left(\begin{array}{c}
t^{n} \\
t^{n-1}
\end{array}\right), \\
& \tilde{H}_{-1}\left(\begin{array}{c}
t^{n} \\
t^{n-1}
\end{array}\right) \cong\left(\begin{array}{c}
t^{n-1} \\
t^{n-2}
\end{array}\right) .
\end{aligned}
$$

Referring to the expressions (48), the three components of the gauge operator $\tilde{H}$ are deduced

$$
\begin{gathered}
\tilde{H}_{1}=\left(\begin{array}{cc}
-4 k^{2} t^{3} \frac{\mathrm{d}^{2}}{\mathrm{~d} t^{2}}-6 k^{2} t^{2} \frac{\mathrm{d}}{\mathrm{d} t}+\left(a_{1}-2 k^{2}\right) t & \theta k^{2} t^{2} \\
\theta & -4 k^{2} t^{3} \frac{\mathrm{d}^{2}}{\mathrm{~d} t^{2}}-18 k^{2} t^{2} \frac{\mathrm{d}}{\mathrm{d} t}+\left(a_{2}-12 k^{2}\right) t
\end{array}\right), \\
\tilde{H}_{0}=\left(\begin{array}{cc}
4\left(k^{2}+1\right) t^{2} \frac{\mathrm{d}^{2}}{\mathrm{~d} t^{2}}+\left(4-4 k^{2}\right) t \frac{\mathrm{d}}{\mathrm{d} t}++1+b+k^{2} & -\theta\left(k^{2}+1\right) t \\
0 & 4\left(k^{2}+1\right) t^{2} \frac{\mathrm{d}^{2}}{\mathrm{~d} t^{2}}+12\left(k^{2}+1\right) t \frac{\mathrm{d}}{\mathrm{d} t}+4+4 k^{2}-b
\end{array}\right) \\
\tilde{H}_{-1}=\left(\begin{array}{cc}
-4 t \frac{\mathrm{d}^{2}}{\mathrm{~d} t^{2}}+\left(2+4 k^{2}\right) \frac{\mathrm{d}}{\mathrm{d} t} & \theta \\
0 & -4 t \frac{\mathrm{d}^{2}}{\mathrm{~d} t^{2}}-6 \frac{\mathrm{d}}{\mathrm{d} t}
\end{array}\right)
\end{gathered}
$$

As it is shown by the relation (48), the above operators $\tilde{H}_{1}, \tilde{H}_{0}$ and $\tilde{H}_{-1}$ are respectively the matrix operators which increases, preserves and reduces the de- 
gree of the generic element $\psi$ given by the Equation (47). As a consequence the vector $\tilde{H} \psi$ can be decomposed as follows

$$
\tilde{H} \psi=\operatorname{diag}\left(\begin{array}{ll}
t^{n+1} & t^{n}
\end{array}\right) M_{1}\left(\begin{array}{l}
\alpha_{0} \\
\beta_{0}
\end{array}\right)+\operatorname{diag}\left(\begin{array}{ll}
t^{n} & t^{n-1}
\end{array}\right) \tilde{M}_{1}\left(\begin{array}{l}
\alpha_{1} \\
\beta_{1}
\end{array}\right)+\operatorname{diag}\left(\begin{array}{ll}
t^{n} & t^{n-1}
\end{array}\right) M_{0}\left(\begin{array}{l}
\alpha_{0} \\
\beta_{0}
\end{array}\right)(50)
$$

where the constant $2 \times 2$-matrices $M_{1}, \tilde{M}_{1}$ and $M_{0}$ can be computed explicitly after a some calculations

$$
\tilde{H}_{1}\left(\begin{array}{c}
\alpha_{0} t^{n} \\
\beta_{0} t^{n-1}
\end{array}\right)=\operatorname{diag}\left(\begin{array}{ll}
t^{n+1} & t^{n}
\end{array}\right) M_{1}\left(\begin{array}{c}
\alpha_{0} \\
\beta_{0}
\end{array}\right)
$$

where

$$
M_{1}=\left(\begin{array}{cc}
-4 k^{2} n(n-1)-6 k^{2} n+a_{1}-2 k^{2} & \theta k^{2} \\
\theta & -4 k^{2}(n-1)(n-2)-18 k^{2}(n-1)+a_{2}-12 k^{2}
\end{array}\right)
$$

One can easily deduce the matrix $\tilde{M}_{1}$ from the following expression

$$
\tilde{H}_{1}\left(\begin{array}{l}
\alpha_{1} t^{n-1} \\
\beta_{1} t^{n-2}
\end{array}\right)=\operatorname{diag}\left(t^{n} t^{n-1}\right) \tilde{M}_{1}\left(\begin{array}{l}
\alpha_{1} \\
\beta_{1}
\end{array}\right),
$$

where

$$
\tilde{M}_{1}=\left(\begin{array}{cc}
-4 k^{2}(n-1)(n-2)-6 k^{2}(n-1)+a_{1}-2 k^{2} & \theta k^{2} \\
\theta & -4 k^{2}(n-2)(n-3)-18 k^{2}(n-2)+a_{2}-12 k^{2}
\end{array}\right)
$$

finally the third matrix $M_{0}$ is easily found as follows

$$
\tilde{H}_{0}\left(\begin{array}{c}
\alpha_{0} t^{n} \\
\beta_{0} t^{n-1}
\end{array}\right)=\operatorname{diag}\left(t^{n} t^{n-1}\right) M_{0}\left(\begin{array}{c}
\alpha_{0} \\
\beta_{0}
\end{array}\right)
$$

where

$$
M_{0}=\left(\begin{array}{cc}
\left(4 k^{2}+4\right) n(n-1)+\left(4-4 k^{2}\right) n+1+b+k^{2} & -\theta\left(k^{2}+1\right) \\
0 & \left(4 k^{2}+4\right)(n-1)(n-2)+\left(12+12 k^{2}\right)(n-1)+4+4 k^{2}-b
\end{array}\right)
$$

Referring to the three QES conditions given by the relations (7) and to the expressions of the previous three $2 \times 2$-matrices $M_{1}, \tilde{M}_{1}$ and $M_{0}$, one can easily compute algebraically the three necessary and sufficient conditions for the gauge Hamiltonian $\tilde{H}$ given by the expressions (46) to be quasi-exactly solvable as follows:

1) the first QES condition is as follows

$$
\begin{aligned}
\operatorname{det} M_{1}=0, & \\
\theta^{2}= & \left(16 n^{4}+32 n^{3}+28 n^{2}+16 n+4\right) k^{2}-2 a_{1}\left(2 n^{2}+3 n+1\right) \\
& -2 a_{2}\left(2 n^{2}+n+1\right)+\frac{a_{1} a_{2}}{k^{2}}
\end{aligned}
$$

2) the second QES condition is easily checked

$$
\begin{gathered}
\operatorname{det} \tilde{M}_{1}=0 \\
a_{1}=\frac{64 k^{2} n^{3}+16 k^{2} n^{2}-48 k^{2} n+12 k^{2}+(8 n-2) a_{2}}{a_{2}-8 n+2}
\end{gathered}
$$


3) finally, the third QES condition is found

$$
b=7 k^{2} n+2 n^{2}+n-\frac{a_{1} k^{2}+a_{1}}{2 k^{2}}+\frac{k^{2}+1}{2}
$$

\section{Conclusion}

In this paper, we have applied the QES analytic method in order to construct a $2 \times 2$-matrix QES Hamiltonian which is associated to a Jacobi elliptic potential. For both two cases considered, $\delta=1$ and $\delta=2$, more precisely, we have computed the three necessary and sufficient algebraic QES conditions for the Jacobi elliptic Hamiltonian to have an invariant vector space.

\section{Acknowledgements}

I thank Pr. Yves Brihaye of useful discussions.

\section{Conflicts of Interest}

The author declares no conflicts of interest regarding the publication of this paper.

\section{References}

[1] Turbiner, A.V. (1988) Quasi-Exactly Solvable Problems and $s l(2)$ Algebra. Communications in Mathematical Physic, 118, 467-474. https://doi.org/10.1007/BF01466727

[2] Ushveridze, A.G. (1995) Quasi-Exactly Solvable Models in Quantum Mechanics. Institute of Physics Publishing, Bristol.

[3] Turbiner, A.V. (1989) Lamé Equation $\operatorname{sl}(2)$ Algebra and Isospectral Deformations. Journal of Physics A: Mathematical and General, 22, 1-144. https://doi.org/10.1088/0305-4470/22/1/001

[4] Shifman, M.A. and Turbiner, A.V. (1989) Quantal Problems with Partial Algebraization of Spectrum. Communications in Mathematical Physics, 126, 347-365. https://doi.org/10.1007/BF02125129

[5] González-López, A., Kamran, N. and Olver, P.J. (1993) Normalizability of One-Dimensional Quasi-Exactly Solvable Shrödinger Operators. Communications in Mathematical Physics, 153, 117-146. https://doi.org/10.1007/BF02099042

[6] González-López, A, Kamran, N. and Olver, P.J. (1991) Quasi-Exactly Solvable Lie Algebras of Differential Operators in Two Complex Variables. Journal of Physics $A$, 24, 3995. https://doi.org/10.1088/0305-4470/24/17/016

[7] Zhdanov, R. (1997) Quasi-Exactly Solvable Matrix Models. Physics Letters B, 405, 253-256. https://doi.org/10.1016/S0370-2693(97)00655-2

[8] Brihaye, Y. and Kosinski, P. (1997) Quasi-Exactly Solvable Matrix Models in sl( $n)$. Physics Letters $B, 424,43-47$. https://doi.org/10.1016/S0370-2693(98)00167-1

[9] Brihaye, Y. and Hartmann, B. (2001) Quasi-Exactly Solvable $N \times N$-Matrix Shrödinger Operators. Modern Physics Letters A, 16, 1895-1906. https://doi.org/10.1142/S0217732301005242 
[10] Brihaye, Y., Nininahazwe, A. and Prasad Mandal, B. (2007) PT-Symmetric, Quasi-Exactly Solvable Matrix Hamiltonians. Journal of Physics A: Mathematical and Theoretical, 40, 13063-13073. https://doi.org/10.1088/1751-8113/40/43/014

[11] Nininahazwe, A. (2013) Matrix Quasi-Exactly Solvable Jacobi Elliptic Hamiltonian. Open Journal of Microphysics (OJM), 3, 53-59. https://doi.org/10.4236/ojm.2013.33010

[12] Nininahazwe, A. (2018) Non-Hermitian Matrix Quasi-Exactly Solvable Hamiltonian. Open Journal of Microphysics (OJM), 8, 15-25. https://doi.org/10.4236/ojm.2018.83003

[13] Nininahazwe, A. (2020) PT-Symmetric Matrix Quasi-Exactly Solvable Razhavi Potential. Open Journal of Microphysics (OJM), 10, 9-20.

https://doi.org/10.4236/ojm.2020.102002 